\title{
Coherent Chromatic Effect in the Transmission Electron Microscope
}

\author{
Rolf Erni \\ Electron Microscopy Center, Empa-Swiss Federal Laboratories for Materials Science and Technology, \\ CH-8600 Dübendorf, Switzerland
}

(Received 5 October 2015; revised manuscript received 7 January 2016; published 17 March 2016)

\begin{abstract}
Under the assumption of local atomic scattering, elastic electron scattering at finite scattering angles implies a small but finite energy loss. This energy loss, which under conventional imaging conditions in high-resolution transmission electron microscopy is of the order of $0.1 \mathrm{meV}$ and thus negligible, increases by more than 2 orders of magnitude if light elements are investigated at sub-Ångström resolution. For a microscope of finite chromatic aberration, the energy loss leads to an element-specific chromatic effect which increases with the instrument resolution and with decreasing mass of the scattering atom. Despite that this effect is small, it can degrade the achievable image contrast. However, the effect can be considered in the optimization of the phase-contrast imaging conditions and even be beneficial to enhance the relative image contrast of light atoms in the presence of heavy atoms.
\end{abstract}

DOI: 10.1103/PhysRevLett.116.116101

The most common image formation mechanism in highresolution transmission electron microscopy (HRTEM) is based on the elastic interaction of electrons with atoms. A broad, partially coherent electron beam illuminates an electron transparent specimen. On transmitting the specimen the electrons are scattered by the atoms in the specimen and thus carry at the exit-plane of the specimen structural information which is then translated into a projection image of the specimen. Elastic electron scattering is a coherent process which implies a distinct phase relation between the incoming and outgoing electron. The coherence of the scattering process leads to diffraction, which for crystalline specimens limits the allowed scattering vectors to a set that is represented by Bragg reflections in the diffraction plane. Moreover, elastic scattering demands that the inherent energy state of the scattering objects is maintained. For this reason it is generally assumed that the energy of the electron before and after the elastic scattering process is identical. This assumption holds well, in general [1]. However, recent progress in electron optical instrumentation has boosted the imaging performance. Instrument resolutions near and below $50 \mathrm{pm}$ can nowadays be achieved on dedicated aberrationcorrected transmission electron microscopes [2-4] and the trend towards higher resolution and higher sensitivity is unbroken. A condition of the ever increasing resolution demands is that electrons scattered to higher angles need to be transferred to the image plane without being negatively affected by lens aberrations. Along with the increased resolution and the aberration-corrected optics, a demand towards using electrons of lower primary energy has emerged with the aim to minimize knock-on radiation damage in materials which consist of light elements. Instead of using primary electron energies of $200 \mathrm{keV}$ or higher, with the improved optics atomic-resolution imaging is nowadays feasible at electron energies of $50 \mathrm{keV}$ and even lower [5,6]. In order to maintain the resolution at reduced electron energy, higher scattering angles need to be considered in the image formation process. With this technological advancement and future targets [7], previously irrelevant effects have become relevant and even resolution limiting [8]. One aspect that has not been addressed so far is the above-mentioned assumption that the energy of the elastically scattered electron is fully preserved. The present work disregards this approximation and analyzes its impact on the image formation in transmission electron microscopy.

The fact that the momentum and energy transfer by elastic scattering from the impinging electron to an atom in a solid can be of importance is reflected in knock-on radiation damage. A primary electron, which is elastically scattered to high angles or backscattered, can transfer sufficient kinetic energy $(\approx 5-20 \mathrm{eV})$ to permanently displace an atom from its position (see, e.g., Refs. [9,10]). The elastically deposited energy can even lead to the thermal breakdown of the specimen [11]. Moreover, using $30 \mathrm{keV}$ electrons, Boersch et al. [12] succeeded to measure atomspecific energy losses $(0.1-5 \mathrm{eV})$ of electrons scattered elastically to high angles $(\geq \pi / 4)$. The work of Boersch et al. [12] showed that electrons interact with individual atoms and not with the crystal as a whole, at least when elastically scattered to high angles. Nowadays, the energy loss measurable by elastic electron scattering, so-called electron Rutherford backscattering (ERBS), can be used for elemental characterization and isotope discrimination (see, e.g., Refs. $[13,14])$. Knock-on radiation damage, the experiments of Boersch et al. [12], and the feasibility of ERBS provide evidence that elastic electron scattering at high scattering angles is localized at the site of the atom. Should the elastically scattered electrons interact with the 
entire solid, the momentum change of the electron would be transferred to a large mass and the energy transfer would thus be too small to cause knock-on radiation damage or to make it accessible by energy-loss spectroscopy. Hence, for large scattering angles, elastic electron scattering is localized at the site of the atom resulting in an atom-specific electron energy loss that increases with the momentum change of the scattered electron. Assuming that the same localized interaction takes place at small scattering angles relevant for HRTEM (typically $<50 \mathrm{mrad}$ ), the energy transfer and the corresponding energy loss must be small, of the order of $0.1 \mathrm{meV}$ [1]. Moreover, for small scattering angles it is generally assumed that elastic electron scattering is not localized at the site of the atom, but that the electron interacts with the entire crystal, implying that the energy losses are negligible. This assumption is commonly supported by the occurrence of interference phenomena (diffraction). However, considering, as an example, $50 \mathrm{keV}$ electrons undergoing an energy loss of about $100 \mathrm{meV}$, the coherence length $\left(\bar{\lambda}^{2} / \Delta \lambda\right.$, where $\lambda$ is the electron wavelength) would still be in the range of micrometers and thus sufficient to allow for interference effects [15]. Hence, the presence of a small energy loss does not necessarily imply the absence of diffraction. Moreover, a condition for considering electron scattering to be localized at the site of the atom is that $\theta R>\lambda$ [14], where $\theta$ is the (small) scattering angle $(\sin \theta \approx \theta)$ and $R$ an interatomic distance $(R \approx 200 \mathrm{pm})$. Considering a modern low-voltage transmission electron microscope, which enables a resolution of $80 \mathrm{pm}$ at $50 \mathrm{kV}(\theta=67 \mathrm{mrad})$, this condition is essentially fulfilled.

With the new and future demands in HRTEM of enhancing the relevant scattering angles in order to advance the resolution, electron scattering in HRTEM has reached the regime where elastic electron scattering is considered to be localized at the site of the atom. Under this assumption, the impact of small elastic energy losses in HRTEM is assessed by forecasting the corresponding imaging effects. This analysis is of relevance for quantitative image interpretation but also for advancing the understanding of elastic electron scattering. Considering the positive chromatic aberration of a round electron lens [16], electrons, which suffered from a small elastic energy loss, experience a shorter focal length and form a disk of confusion in the image plane. Therefore, for transmission electron microscopes, which are not equipped with a corrector for the chromatic aberration, and whose energy spread of the electron beam is small (monochromated microscopes), the impact of an energy loss, caused by elastic scattering, is not necessarily negligible.

We combine a particle model with wave optics to derive a quantitative description of the above qualitatively discussed effect. The combination of these two concepts is solely justified by the goal of deriving the impact of the elastic energy loss on the image contrast. From the conservation of momentum and energy, the following relation for the energy transferred from the electron of rest mass $m_{e}$ to the atom $i$ of rest mass $m_{i}$ during an elastic scattering event can be derived [17]

$$
\Delta E(\theta)=\frac{2 E_{0}\left(E_{0}+2 m_{e} c^{2}\right)}{m_{i} c^{2}} \sin ^{2} \frac{\theta}{2} .
$$

The primary electron energy is $E_{0}$ and $c$ the speed of light. Integration of $\Delta E$ over the entire solid angle, considering the elastic scattering factor and the atomic density, yields the energy elastically deposited to the solid [11]. On the other hand, Eq. (1) describes the energy loss of an electron which is elastically scattered by an atom $i$ to an angle $\theta$. For small values of $\theta$, the sine-term can be approximated by $\theta^{2} / 4$. The estimated energy losses in conventional HRTEM are typically below $0.1 \mathrm{meV}[1,17]$ and thus irrelevant for the image formation. However, for highest resolution transmission electron microscopes, where large scattering angles $\theta$ need to be considered and which aim at observing light atoms, $\Delta E$ can become of relevance. Considering a carbon atom $\left(m_{\text {carbon }}=2 \times 10^{-26} \mathrm{~kg}\right)$ which scatters a $50 \mathrm{keV}$ electron to a scattering angle to achieve a microscope resolution of $0.8 \AA$, the elastic energy loss $\Delta E$ of the electron exceeds $10 \mathrm{meV}$ and thus is in the range of the energy spread of electron microscopes, which are equipped with modern electron monochromators $[18,19]$. Moreover, the energy loss hardly depends on the primary electron energy $E_{0}$. For scattering angles corresponding to resolutions of $0.5,0.8$, and $1.0 \AA$, the energy losses are 27, 11, and $7 \mathrm{meV}$ for $E_{0}=50 \mathrm{keV}$. Assuming the same instrument resolutions for $E_{0}=300 \mathrm{keV}$, the corresponding energy losses increase by less than $0.1 \%$. In order to evaluate the impact of the elastic energy loss on the image characteristics, the wave aberration $\chi$ can be written as [20]

$\chi(\theta, \Delta E)=\frac{1}{2} C_{1} \theta^{2}+\frac{1}{4} C_{3} \theta^{4}+\frac{1}{6} C_{5} \theta^{6}+\frac{1}{2} \kappa(\Delta E) C_{c} \theta^{2}$.

Only rotationally symmetric aberrations are considered here. The first three terms on the right-hand side are the geometrical aberrations, namely, defocus $C_{1}$ and the spherical aberrations of third $\left(C_{3}\right)$ and fifth $\left(C_{5}\right)$ order. The fourth term in Eq. (2) describes the effect of the chromatic aberration $C_{c}$ which depends on the chromatic parameter $\kappa=\Delta E / E_{0}$. Hence, the last term accounts for the impact of an electron, whose energy deviates by $\Delta E$ from the nominal energy $E_{0}$, and thus describes the focus variation with electron energy [20]. It is further assumed that the electron beam is monochromatic and thus that the instrument-related chromatic effect is negligible. Substitution of $\Delta E$ from Eq. (1) in Eq. (2) allows for eliminating the $\Delta E$ dependence of $\chi$ and yields 


$$
\chi(\theta)=\frac{1}{2} C_{1} \theta^{2}+\frac{1}{4} C_{3} \theta^{4}+\frac{1}{6} C_{5} \theta^{6}+\frac{1}{4} C_{e} \theta^{4}
$$

with

$$
C_{e}=\frac{E_{0}+2 m_{e} c^{2}}{m_{i} c^{2}} C_{c} .
$$

The $C_{e}$ term describes the coherent chromatic defocus offset of elastically scattered electrons. As $C_{e}$ relates to a chromatic effect of coherently (i.e., elastically) scattered electrons, it can be understood as a coherent chromatic effect. It can be seen that a (positive) third-order spherical aberration $C_{3}$ and the $C_{e}$ term both have the same angular dependence and essentially cause the same qualitative effect. Both (positive) $C_{3}$ and $C_{e}$ cause electrons, which are scattered to a finite angle $\theta$, to be focused in front of the Gaussian image plane. Hence, the factor $C_{e}$ could be interpreted as an offset of the third-order spherical aberration $C_{3}$. However, Eq. (4) reveals that, unlike $C_{3}, C_{e}$ is not an instrumental optical constant; $C_{e}$ depends on the mass $m_{i}$ of the atom that scatters the electron. The impact of the $C_{e}$ term increases with increasing constant of chromatic aberration $C_{c}$ and with decreasing mass $m_{i}$ of the scattering atom. Moreover, unlike the common chromatic effects in HRTEM, $C_{e}$ slightly increases with increasing $E_{0}$. Considering electrons scattered at carbon atoms and a $C_{c}$ of $2 \mathrm{~mm}, C_{e}$ increases from 191 to $235 \mathrm{~nm}$ if the primary electron energy $E_{0}$ is increased from 50 to $300 \mathrm{keV}$. Despite that this value is small, and probably at the edge of the current precision by which $C_{3}$ can be measured, we nevertheless analyze its impact on the imaging characteristics.

Simulations were performed assuming an aberrationcorrected $50 \mathrm{kV}$ microscope with $C_{c}$ of $2 \mathrm{~mm}$ and an information limit of $0.8 \AA$, corresponding to a maximum scattering angle $\theta$ of $67 \mathrm{mrad}$, with adjustable $C_{1}, C_{3}$, and $C_{5}$. This corresponds to a modern transmission electron microscope with an aberration corrector that allows for optimizing fifth-order aberrations (see, e.g., Ref. [21]). As the effect increases with decreasing mass of the scattering atom, simulations were carried out for graphene for which the $C_{e}$ parameter amounts to $0.2 \mu \mathrm{m}$. The microscope shall be set up for optimal negative phase contrast [22] yielding for $C_{1}, C_{3}$, and $C_{5}+4.5 \mathrm{~nm},-4.0 \mu \mathrm{m}$, and $+0.8 \mathrm{~mm}$, respectively. Figure 1 shows two line profiles of the calculated images of two neighboring carbon atoms in graphene. One profile is based on a simulation without considering $C_{e}$ (full line) while the other profile takes the $C_{e}$ effect as an offset on $C_{3}$ into account (dashed line). The line profiles reveal that the effect of $C_{e}$ leads to a reduction of the peak contrast of about $7 \%$. The contrast reduction of $7 \%$ for a current state-of-the-art low-voltage microscope might be difficult to verify. However, for future transmission electron microscopes of enhanced resolution the effect is strongly amplified. For a $50 \mathrm{kV}$ microscope of $0.5 \AA$ resolution the contrast reduction of carbon atoms due

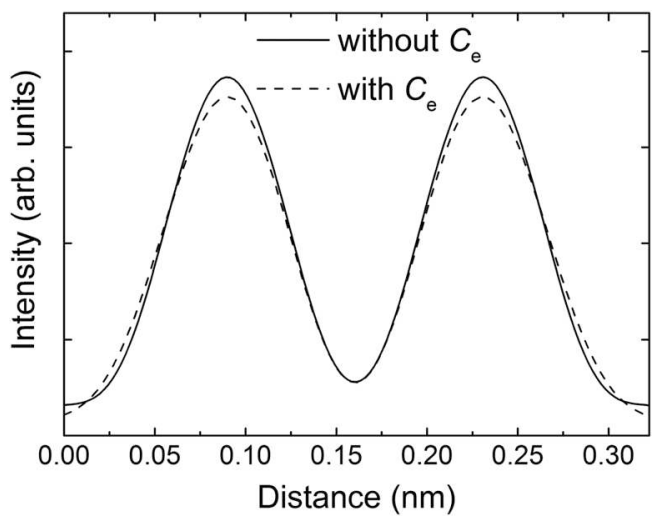

FIG. 1. Calculated intensity line profiles of two neighboring carbon atoms in graphene for a $50 \mathrm{kV}$ microscope of $0.8 \AA$ information transfer under optimized negative phase contrast conditions. The full line shows the intensity without considering $C_{e}$, while the dashed line includes a $C_{e}$ effect of $0.2 \mu \mathrm{m}$.

to $C_{e}$ amounts to about 50\%; see Fig. 2. Importantly, a suitable adjustment of $C_{3}$ could compensate for the contrast reduction due to $C_{e}$. However, as $C_{e}$ depends on $m_{i}$, this adjustment is only feasible if only one element is present.

Figure 3 shows an atomic model (a) and simulations (b)-(d) for a graphene sample which contains a gold substitutional atom [23]. The simulations were carried out based on an independent atom model for a $50 \mathrm{kV}$ microscope of $0.8 \AA$ resolution. The simulation in Fig. 3(b)

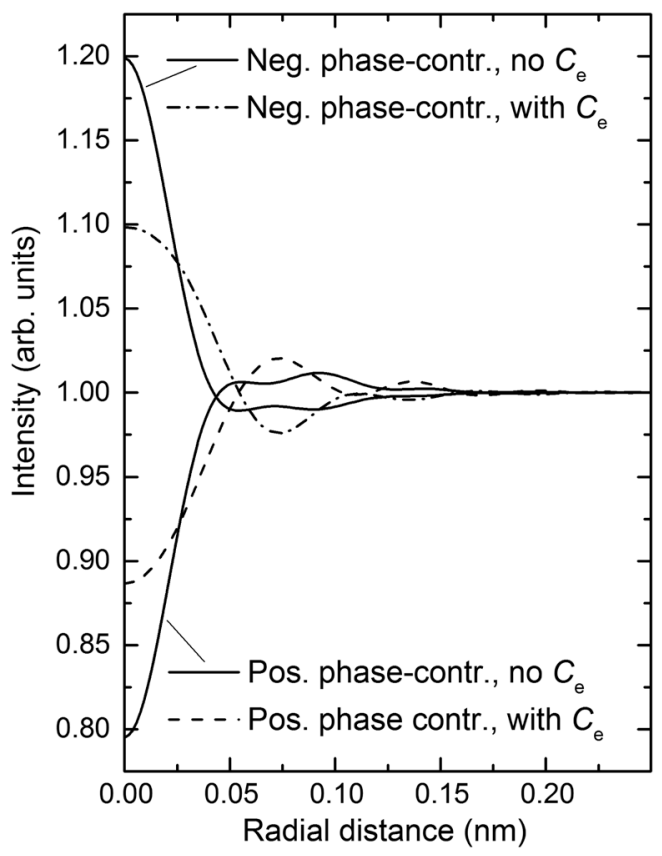

FIG. 2. Radial intensity profiles of calculated phase-contrast images of carbon atoms. The $C_{i}$ were calculated for optimal negative and positive phase contrast according to Lentzen [22] for a $50 \mathrm{kV}$ microscope of $0.5 \AA$ information transfer; $C_{1}= \pm 1.75 \mathrm{~nm}, C_{3}=\mp 610 \mathrm{~nm}$, and $C_{5}= \pm 46.4 \mu \mathrm{m}$. Adding the effect of $C_{e}(0.5 \mu \mathrm{m})$ reduces the image contrast of the carbon atom for both positive and negative phase contrast by about $50 \%$. 


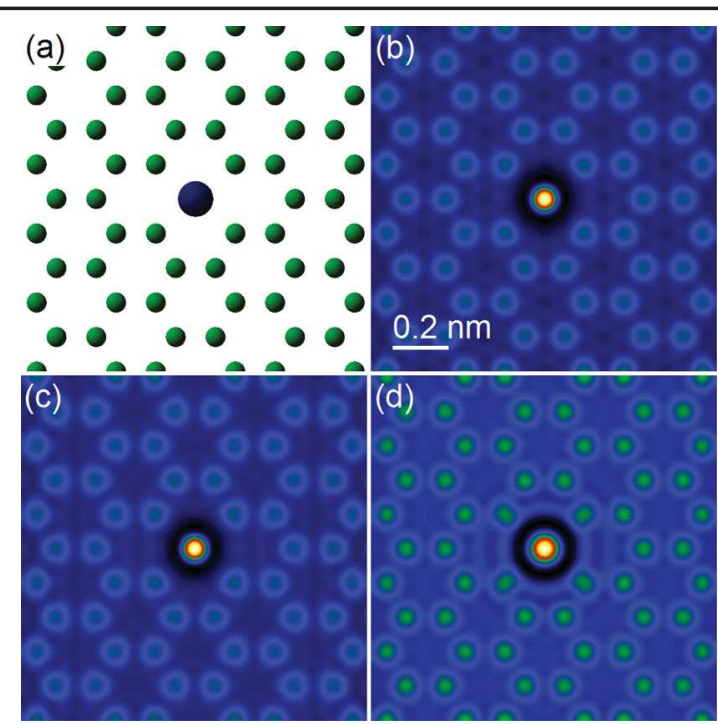

FIG. 3. (a) Atomic model used for the simulations; Au atom (large, blue) and carbon atoms (small, green). (b) Simulated image for optimal negative phase contrast which does not consider $C_{e}$. (c) Simulated image for optimal negative phase contrast which takes the $C_{e}$ effect into account. (d) Simulated image for optimized imaging conditions for carbon atoms when considering the $C_{e}$ effect (see text). The images are displayed on a temperature color scale which, in order to visualize the relative image contrast of carbon and the gold atoms, is normalized to the peak intensity of $\mathrm{Au}$ atoms.

does not consider the effect of $C_{e}$, while Fig. 3(c) takes $C_{e}$ into account. A small reduction in contrast of the carbon atoms in Fig. 3(c) is observable, as already documented in Fig. 1. As the $C_{e}$ values for gold and carbon are different (11 nm for Au vs $191 \mathrm{~nm}$ for C), $C_{3}$ cannot be adjusted to correct for the element-specific $C_{e}$ effect. On the other hand, as $C_{e}$ results in element specific imaging characteristics, it can be used to enhance the contrast of light elements in the presence of heavy ones. This is demonstrated in Fig. 3(d). The imaging conditions were adjusted such that together with the preset $C_{3}$ value the offset due to $C_{e}$ results in optimal imaging conditions for carbon atoms. This, of course, implies that the $C_{3}$ value is suboptimal for imaging the $\mathrm{Au}$ atom. By interference of the spherical waves emerging from the gold and the carbon atoms, the suboptimal imaging condition of the Au atom leads to an apparent elongation of the adjacent carbon atoms. For the case shown in Fig. 3(d) the peak intensity of the carbon atoms can be enhanced by about $20 \%$ compared to the peak intensity of the $\mathrm{Au}$ atom. This relative contrast enhancement is clearly visual when comparing Fig. 3(c) with 3(d) and it could already be experimentally verified on presentday microscopes provided the imaging parameters can be set with sufficient precision.

Another, however future, possibility of exploring the effect of $C_{e}$ concerns the case where the elastic energy loss and the corresponding effect due to $C_{e}$ is used for discriminating different isotopes.
Experimental verification of the $C_{e}$ effect is possible, but might still be out of reach with the current generation of electron microscopes. The effect of $C_{e}$ could be measured by precisely assessing the (effective) $C_{3}$ on two different (thin) samples, one consisting of heavy elements and one consisting of a light element, using a geometrical (but not chromatic) aberration-corrected transmission electron microscope. Should the effect of $C_{e}$ be of significance, the measurement of the effective $C_{3}$ on the light sample would lead to a slightly larger value. However, as the $C_{e}$ effect emerges (isotropically) in the sample, while the Zemlin tableau assesses the optical behavior of the instrument as a function of beam tilt, the effective $C_{3}$ cannot be measured by the Zemlin tableau method. Nevertheless, the effective $C_{3}$ could be determined based on the image intensity (see, e.g., Ref. [24]) as outlined in Fig. 3. For a reliable assessment of $C_{e}$, the precision of the measurement would need to be of the order of a few tens of nanometers or better. Currently, this is very difficult to achieve, especially regarding the limited lifetime of the optical state of state-of-the-art microscopes [25]. Moreover, incoherent inelastic scattering contributions can affect the image contrast. Because of their incoherent nature they do not contribute to $C_{e}$, but can impair the precision by which the effective $C_{3}$ can be measured. An alternative method to indirectly verify the effect of $C_{e}$ is to measure the energy loss of Bragg diffracted beams, similar to the experiments of Boersch et al. [12], but for small scattering angles. In order to reliably measure angle-dependent energy losses of the order of $10 \mathrm{meV}$, a monochromated microscope of high energy resolution would be required $[18,19]$.

Under the assumption that elastic electron scattering is localized at the site of the atom, we can conclude the following points. Even when considering elastic electron scattering only, an ideal monochromatic and a chromatic aberration-corrected microscope are not equivalent as the latter does not show the $C_{e}$ effect. Regarding the wave aberration function, $C_{e}$ has the same angular dependence as the third-order spherical aberration $C_{3}$. Unlike $C_{3}, C_{e}$ depends on the mass of the atom that is involved in the scattering process. Therefore, $C_{e}$ can be fully compensated by a suitable choice of $C_{3}$, but only for the case that there is one atomic species present in the sample. As the $C_{e}$ effect increases with decreasing atomic mass, a suitable choice of the isotropic aberration constants $\left(C_{1}, C_{3}\right.$, and $\left.C_{5}\right)$ allows for element-specific enhancement of the relative image contrast of light elements. For multiple elastic scattering in samples of finite thickness, the energy losses of the individual elastic scattering events add up and the angular dependence of the energy loss is not describable by Eq. (1) anymore. The coherent chromatic effect $C_{e}$ is small, but it might be of relevance for future aberration-corrected electron microscopes with $C_{c} \neq 0$, which aim for deep sub-Ångström resolution particularly at low acceleration voltages. 
The author would like to thank Dr. Heiko Müller and Dr. Peter Hartel (CEOS GmbH), Dr. Juri Barthel (Ernst Ruska-Centre, Jülich) and Professor Bernard Jouffrey for valuable comments on the manuscript and for sharing their expertise.

* Corresponding author. rolf.erni@empa.ch

[1] B. Jouffrey, in Cours de l'École de Microscopie Électronique en Science des Matériaux, edited by B. Jouffrey, A. Bourret, and C. Colliex (CNRS, Paris, 1983), Chap. 7, p. 85.

[2] R. Erni, M. D. Rossell, C. Kisielowski, and U. Dahmen, Phys. Rev. Lett. 102, 096101 (2009).

[3] H. Sawada, N. Shimura, F. Hosokawa, N. Shibata, and Y. Ikuhara, Microscopy 64, 213 (2015).

[4] T. Akashi, Y. Takahashi, T. Tanigaki, T. Shimakura, T. Kawasaki, T. Furutsu, H. Shinada, H. Müller, M. Haider, N. Osakabe, and A. Tonomura, Appl. Phys. Lett. 106, 074101 (2015).

[5] U. Kaiser, J. Biskupek, J. C. Meyer, J. Leschner, L. Lechner, H. Rose, M. Stöger-Pollach, A. N. Khlobystov, P. Hartel, H. Müller, M. Haider, S. Eyhusen, and G. Benner, Ultramicroscopy 111, 1239 (2011).

[6] H. Sawada, T. Sasaki, F. Hosokawa, and K. Suenaga, Micron 63, 35 (2014).

[7] S. J. Pennycook and S. V. Kalinin, Nature (London) 515, 487 (2014).

[8] S. Uhlemann, H. Müller, P. Hartel, J. Zach, and M. Haider, Phys. Rev. Lett. 111, 046101 (2013).

[9] A. Zobelli, A. Gloter, C. P. Ewels, G. Seifert, and C. Colliex, Phys. Rev. B 75, 245402 (2007).
[10] A. Weickenmeier and H. Kohl, Acta Crystallogr. Sect. A 47, 590 (1991).

[11] B. Jouffrey and M. Karlík, Microsc., Microanal., Microstruct. 3, 243 (1992).

[12] H. Boersch, R. Wolter, and H. Schoenbeck, Z. Phys. 199, 124 (1967).

[13] M. Vos, E. Weigold, and R. Moreh, J. Chem. Phys. 138, 044307 (2013).

[14] M. Vos, R. P. McEachran, E. Weigold, and R. A. Bonham, Nucl. Instrum. Methods Phys. Res., Sect. B 300, 62 (2013).

[15] M. Born and E. Wolf, Principles of Optics, 7th ed. (Cambridge University Press, Cambridge, England, 1999).

[16] O. Scherzer, Z. Phys. 101, 593 (1936).

[17] L. Reimer and H. Kohl, Transmission Electron Microscopy (Springer, New York, 2008).

[18] O. L. Krivanek, T. C. Lovejoy, N. Dellby, T. Aoki, R. W. Carpenter, P. Rez, E. Soignard, J. Zhu, P. E. Batson, M. J. Lagos, R. F. Egerton, and P. A. Crozier, Nature (London) 514, 209 (2014).

[19] L. H. G. Tizei, Y. C. Lin, M. Mukai, H. Sawada, A. Y. Lu, L. J. Li, K. Kimoto, and K. Suenaga, Phys. Rev. Lett. 114, 107601 (2015).

[20] D. Typke and K. Dierksen, Optik (Stuttgart) 99, 155 (1995).

[21] T. Sasaki, H. Sawada, F. Hosokawa, Y. Kohno, T. Tomita, T. Kaneyama, Y. Kondo, K. Kimoto, Y. Sato, and K. Suenaga, J. Electron Microsc. 59, S7 (2010).

[22] M. Lentzen, Microsc. Microanal. 14, 16 (2008).

[23] S. Stolbov and M. Alcàntara Ortigoza, J. Chem. Phys. 142, 154703 (2015).

[24] C. L. Jia, J. Barthel, F. Gunkel, R. Dittmann, S. HoffmannEiffert, L. Houben, M. Lentzen, and A. Thust, Microsc. Microanal. 19, 310 (2013).

[25] J. Barthel and A. Thust, Ultramicroscopy 134, 6 (2013). 